\title{
Estudo da prevalência de afecções de cavidade oral em equídeos de matadouro*
}

\section{Prevalence study of oral cavity affections in abattoir's equidae}

\author{
Felipe Berbari Neto, ${ }^{* *}$ Paulo Roberto da Cruz Reibolt, ${ }^{* * *}$ Domingos Cachineiro Rodrigues Dias, ${ }^{* \star * *}$ \\ Cinthya Dessaune Neves, ${ }^{* * * *}$ Eduardo Mitke Brandão Reis, ${ }^{* *}$ Gabriel de Faria Pereira***
}

\begin{abstract}
Resumo
Um total de 423 cavidades orais de equinos sem raça específica e com idade média de 9,4 anos foram avaliadas através de inspeção post mortem em abatedouro da cidade de Araguari, estado de Minas Gerais, com o objetivo de verificar a prevalência de 20 alterações orodentais predeterminadas baseando-se na literatura especializada consultada. Dos equídeos avaliados, $99 \%$ foram identificados como portadores de desordens orodentais. A desordem mais prevalente foram as pontas excessivas de esmalte dentário, com 355 ocorrências, a palatite em 280 animais e lesões na mucosa jugal presentes em 135 cavidades orais. Tais números corroboram a importância desse tipo de lesões para os equídeos de uma forma geral, sendo necessários mais estudos com o objetivo de determinar o impacto dessas alterações em animais vivos.
\end{abstract}

Palavras-chave: abatedouro, alterações intraorais, equídeos, odontologia.

\begin{abstract}
A total of 423 oral cavities of post-mortem equidae with no specified breed and an avarage of 9,4 years have been evaluated in an abbatoir in the town of Araguari, state of Minas Gerais in order to verify the prevalence of 20 pre-selected orodental alterations based on the literature review. It was possible to identify orodental disorders in $99 \%$ of the studied equides. The most prevalent disorders were sharp enamel points, with 355 occurrences; palatitis, found in 280 animals and lesions in the jugal mucosa, present in 135 oral cavities. These numbers confirm the importance of this type of injury to horses in general, but more research is needed in order to determine the impact of these injuries in living equines.
\end{abstract}

Keywords: abattoir, intra-oral injuries, equidae, odontology.

\section{Introdução}

As condições ambientais impostas pelo homem, principalmente dieta e confinamento, contrárias à fisiologia e anatomia normal da espécie, são os principais fatores para o desenvolvimento de afecções dentárias nos equídeos. Trata-se de processo contínuo, sendo possível afirmar que a maioria destes animais desenvolverá alguma delas ao longo da vida. O animal se adapta e passa a conviver com as constantes alterações em sua arcada dentária até alcançar um quadro grave com consequências sistêmicas e irreversíveis. Deve-se lembrar de que a cavidade oral do equídeo é profunda, escura, de difícil acesso e que, na maioria das vezes, o mesmo não colabora para que o exame clínico seja feito adequadamente, o que culmina por propiciar o agravamento das alterações (Pagliosa et al., 2006). Dada esta dificuldade física de acesso, o exame clínico minucioso da cavidade oral do equídeo em vida requer equipamentos apropriados e, por vezes, especialização técnica. O exame post mortem veio como método facilitador para avaliação da cavidade oral.

Anormalidades de desenvolvimento dentário ocorrem de forma relativamente comum no cavalo e resultam em ampla gama de apresentações clínicas. Problemas congênitos e/ ou do desenvolvimento presentes durante a erupção dentária podem originar, posteriormente, alterações dentária quando no momento de desgaste. Consequentemente, várias anormalidades dentárias diferentes, cujas patogêneses possam estar inter-relacionadas ou não, comumente estão presentes na hora da apresentação clínica (Easley, 2006).

Cavalos podem adquirir fraturas nos dentes através de muitos mecanismos, incluindo traumas externos, como resultado de coices e mordidas em objetos inanimados (Dixon et al., 2006).

As ondas são desgastes irregulares que formam depressões e relevos recíprocos na superfície oclusal das arcadas opostas

\footnotetext{
*Recebido em 23 de julho de 2013 e aceito em 9 de janeiro de 2014.

**Universidade Federal do Acre, AC.

***Universidade do Grande Rio, RJ.

**** Universidade Federal da Bahia, BA.

${ }^{* * * * *}$ Faculdade de Castelo, ES.

Autor para correspondência: berbarineto@hotmail.com.
} 
(Baker, 2002). As ondas são atribuídas ao retardo da erupção de um ou mais dentes decorrente da retenção de dentes decíduos - capas - ou de perdas dentárias (Pagliosa et al., 2006).

$\mathrm{O}$ degrau pode ocorrer em qualquer dente que se torna mais elevado em relação aos adjacentes, devido à falta de desgaste, sendo uma consequência frequente de perda ou extração de um dente oposto (Mueller, 1991). A retenção de dentes decíduos, associada ou não a doença periodontal, pode ser um fator predisponente adicional à ocorrência do degrau (Pagliosa et al., 2006).

O gancho ocorre mais frequentemente na superfície rostral dos segundos pré-molares maxilares e na superfície caudal dos terceiros molares mandibulares, podendo ter outras localizações (Pagliosa et al., 2006). Sua ocorrência é atribuída ao contato oclusal incompleto (Baker, 2002).

Pontas excessivas de esmalte dentário (PEED) são projeções afiadas que geralmente formam-se na borda bucal da superfície oclusal dos dentes pré-molares e molares maxilares (arcada superior) e na borda lingual da superfície oclusal dos dentes pré-molares e molares mandibulares (arcada inferior). Isso ocorre devido à anisognatia, aumentando o ângulo de oclusão e alterando a biomecânica mastigatória (Lane, 1994; Peters et al., 2006), e principalmente devido às alterações na dinâmica mastigatória dos equinos determinadas no processo de domesticação da espécie (Baxter, 2002).

O objetivo deste trabalho foi identificar alterações odontológicas em equídeos post mortem, com intuito de facilitar o exame clínico e disponibilizar uma referência científica válida sobre o tema.

\section{Material e métodos}

O experimento foi realizado em um matadouro de equídeos localizado na cidade de Araguari - MG. No período experimental de três semanas foram realizados sete abates, totalizado 684 animais abatidos. Desse total, 423 cabeças (cavidades orais) foram aleatoriamente analisadas.

Para a coleta de dados foi utilizada uma planilha contendo 20 afecções pré-selecionadas. Para complementar a documentação foi utilizada uma câmera fotográfica exclusivamente para registro e análise das lesões.

Na planilha foram assinaladas todas as ocorrências, além de idade e sexo dos animais. Os seguintes fatores não foram levados em consideração: raça, tipo de alimentação, manejo e história clínica dos animais. A idade foi estabelecida pela cronologia e anatomia dentária conforme Sisson e Grossman (1986). Nos animais com idade superior a cinco anos, o sexo foi estabelecido pela ausência dos caninos (Easley, 2006).

O material foi colhido e analisado no corredor de acesso à seção de graxaria. As cabeças chegavam dentro de um carrinho, de onde eram retiradas e tinham as cavidades orais avaliadas. Em seguida, as amostras eram colocadas num outro carrinho que seguia para a graxaria de modo a não interromper ou atrasar o abate diário.

No processo de abate as cabeças foram partidas por machados pneumáticos. Em algumas delas o processo causou fraturas na região do mento, o que impossibilitou a análise de braquignatismo e prognatismo.

\section{Resultados e discussão}

Do total de animais analisados, em relação ao sexo, $56 \%$ foram identificados como machos, $26 \%$ como fêmeas e $18 \%$ não tiveram o sexo identificado por se tratarem de animais com menos de cinco anos.

Acredita-se que o maior número de machos (56\%) deve-se ao fato de que as fêmeas possuem um valor de mercado como receptoras de embriões maior do que o seu peso em carne.

Em relação às alterações congênitas (Tabela 1), o achado mais comum foi diastema (18\%), seguido por apinhamento $(5,9 \%)$, oligondontia $(4 \%)$ e poliodontia $(0,2 \%)$.

Tabela 1: Alterações odontológicas congênitas em equídeos de matadouro

\begin{tabular}{lcc}
\hline Alterações Congênitas & Valores Absolutos & Porcentagem \\
\hline Diastema & 76 & 18,0 \\
Apinhamento & 25 & 05,9 \\
Oligodontia & 17 & 04,0 \\
Poliodontia & 01 & 00,2 \\
\hline
\end{tabular}

Das afecções aqui apresentadas como congênitas, o diastema é a afecção que possui consequência mais grave de acordo com Carmalt (2003), que é a periodontite nos dentes posteriores. Entretanto, esta relação não foi observada neste experimento. Ainda é possível um animal possuir múltiplos diastemas, aumentando a possibilidade de desenvolver alguma alteração no periodonto. Hole e Dixon (2008) relataram num estudo retrospectivo a incidência de apenas $7 \%$ de diastema. Tal discrepância pode ser justificada, pois a população estudada em abatedouro é constituída por animais que não foram submetidos a uma rotina de exames e tratamentos odontológicos.

Ao contrário do diastema, o apinhamento ou "misplaced tooth" é o congestionamento de dentes e foi observado em aproximadamente $6 \%$ dos animais avaliados (Johnson, 2006). Não foi possível determinar os tipos de oligodontias, que poderiam ser por ausência congênita, retenção de dente no interior da arcada ou oligodontia adquirida (Easley, 2006). Na única arcada com poliodontia, não foi possível afirmar qual era o dente supranumerário. Easley (2006) já havia observado esta dificuldade, dada a semelhança dos dentes, a não ser quando estes são rudimentares ou dismórficos. De acordo com Dixon (2011a), as alterações dentárias congênitas, apesar de poderem ser encaradas como enfermidades dentárias primárias, muitas vezes são apenas manifestações de uma anormalidade inicialmente dos ossos do esqueleto craniofacial. Porém, tais alterações ósseas não foram avaliadas neste experimento, dada a necessidade de não interromper a rotina de abate.

Em relação às alterações de desgaste dentário (Tabela 2), as mais comuns foram as pontas excessivas de esmalte dentário (PEED) $(83,9 \%)$. Esse dado concorda com os achados de Rizzo et al. (2011) que determinaram em cavalos adultos que as principais alterações encontradas no exame odontológico com animais sedados foram as PEED, numa porcentagem de $96 \%$ na ocorrência dessas alterações. Além disso, Hole e Dixon 
(2008) reportaram uma incidência de 100\% de PEED em animais examinados entre os anos de 1999 e 2006, o que reforça a importância dessa alteração dentária na espécie equina.

De acordo com Pagliosa et al. (2006), o processo de domesticação e confinamento que determinou mudança nos hábitos alimentares dos equinos é a maior influência para a alta ocorrência da PEED, o que concorda com a afirmação de Baxter (2002) que considera a adição de alimento concentrado e a menor oferta de forragem como responsáveis pela diminuição do tempo de ingestão e do estímulo a movimentos mastigatórios mais verticais, promovendo não somente a PEED, mas outras alterações no desgaste dentário.

O desnivelamento anterior se mostrou com valores aproximados aos de degraus, ganchos e ondas, corroborando a afirmativa de Rucker (1996), em que incisivos inclinados podem ocorrer quando anormalidade de algum dente posterior está presente, inibindo a excursão e mastigação bilateral.

As outras alterações observadas foram degraus $(29,8 \%)$, seguido por ganchos $(28,4 \%)$, ondas $(23,9 \%)$, desnivelamento anterior $(23,2 \%)$ e fraturas $(5 \%)$ (Tab 2).

Lima et. al. (2011) relataram achados divergentes com os resultados observados desse estudo. Esses autores reportaram uma porcentagem de $65 \%$ na incidência de ganchos dentários em cavalos militares e correlacionaram essa alteração com a incidência de sobremordida.

Tabela 2: Alterações do desgaste dentário em equídeos de matadouro

\begin{tabular}{|c|c|c|}
\hline $\begin{array}{l}\text { Alterações do desgaste } \\
\text { dentário }\end{array}$ & Valores Absolutos & $\begin{array}{c}\text { Porcentagem } \\
(\%)\end{array}$ \\
\hline PEED $^{1}$ & 355 & 83,9 \\
\hline Degrau & 126 & 29,8 \\
\hline Onda & 101 & 23,9 \\
\hline Desnivelamento Anterior & 98 & 23,2 \\
\hline Fraturas & 21 & 5,0 \\
\hline Ganchos $^{2}$ & 120 & 28,4 \\
\hline 106 & 10 & 2,4 \\
\hline 206 & 24 & 5,7 \\
\hline 306 & 24 & 5,7 \\
\hline 406 & 14 & 3,3 \\
\hline 111 & 10 & 2,4 \\
\hline 211 & 14 & 3,3 \\
\hline 311 & 14 & 3,3 \\
\hline 411 & 10 & 2,4 \\
\hline
\end{tabular}

${ }^{1}$ PEED = Pontas excessivas de esmalte dentário. ${ }^{2}$ Sistema Triadan.

O desnivelamento anterior se mostrou com valores percentuais $(23,2 \%)$ aproximados aos de degraus $(29,8 \%)$, ganchos $(28,4 \%)$ e ondas $(23,9 \%)$, corroborando a afirmativa de Rucker (1996) que afirmou que os incisivos inclinados podem se desenvolver quando alguma anormalidade de algum dente posterior está presente, inibindo a excursão e mastigação bilateral simétrica. A distribuição das alterações de desgaste dentária de acordo com o sistema de classificação e identificação dentária de Triadan (Dixon, 2002) demonstra maior incidência dos ganchos rostrais em comparação com os ganchos caudais.
Os animais analisados provavelmente possuíam menos predisposição ao desenvolvimento de ganchos, pois tudo indica que os mesmos eram criados soltos, uma vez que não há, no Brasil, criação destinada à produção de carne (Esalq, 2006), e os animais que vão ao descarte são zootecnicamente inferiores. Animais criados em cocheiras se alimentam acima do nível do solo, o que, segundo Pagliosa et al. (2006), faz com que os mesmos adotem um padrão mastigatório em desacordo com hábito natural de ingestão de alimentos pela espécie, favorecendo a formação de ganchos.

Em relação às fraturas dentárias, a porcentagem de $5 \%$ observada nos animais de abatedouro foi conflitante com o relatado por Farias et al. (2010) que determinaram uma porcentagem de $30 \%$ na ocorrência dessas lesões, sendo a maioria nos dentes molares, trabalhando com equinos atletas da modalidade de polo equestre. Os autores justificam essa alta ocorrência pelo manejo intensivo ao qual o cavalo de esporte é submetido, contrariamente ao que se acredita ser a origem dos animais deste experimento, como citado no parágrafo anterior.

A lesão adquirida em tecidos moles intraorais mais comum foi edema de palato $(66,2 \%)$, seguido por lesão na mucosa jugal $(31,9 \%)$, lesão no palato $(23,6 \%)$ e lesão na língua $(8,7 \%)$, como pode ser observado na Tabela 3.

Tabela 3: Lesões adquiridas em tecidos moles intraorais de equídeos de matadouro

\begin{tabular}{lcc}
\hline Tecidos Moles Intraorais & Valores Absolutos & Porcentagem \\
\hline Edema de palato & 280 & 66,2 \\
Lesões na mucosa jugal & 135 & 31,9 \\
Lesões no palato & 100 & 23,6 \\
Lesões na língua & 37 & 08,7 \\
\hline
\end{tabular}

A ocorrência de edema de palato (palatite) se deve à ingestão de alimentos abrasivos que lesam de forma gradativa o palato duro, causando a sua tumefação, além de ser resposta fisiológica à erupção dentária (Thomassian, 1990). O edema da mucosa do palato também pode ser causado pelo desenvolvimento de outras doenças "dentárias" (Baker, 2002), como as alterações do desgaste, que inicialmente formariam edema na mucosa jugal e por ação da gravidade, edema de palato.

$\mathrm{Na}$ Tabela 4 observa-se que a doença intraoral adquirida mais comum foi o tártaro $(24,1 \%)$, seguido pela periodontite $(9,9 \%)$ e cárie $(3,8 \%)$.

Tabela 4: Doenças dentárias adquiridas em equídeos de matadouro

\begin{tabular}{lcc} 
Doenças Dentárias Adquiridas & Valores Absolutos & Porcentagem \\
\hline Tártaro & 102 & 24,1 \\
Periodontite & 42 & 09,9 \\
Cárie & 16 & 03,8 \\
\hline
\end{tabular}

A formação de tártaro, segundo Wintzer (1990), é um fator que predispõe ao aparecimento de doença periodontal. Os animais avaliados mostraram-se pouco susceptíveis às cáries, o que presumidamente está relacionado com a dieta dos mesmos, 
pobre em carboidratos. De acordo com Dixon (2011b), as cáries são resultantes da fermentação de carboidratos da dieta pelas bactérias orais, levando ao ataque ácido e descalcificação das substâncias inorgânicas do dente. Já a periodontite, além de estar relacionada com o tártaro, ainda possui outras causas (Wintzer, 1990).

\section{Conclusões}

As alterações dentárias mostraram-se importantes nos equinos de abatedouro avaliados, tendo-se em vista que $99 \%$

\section{Referências}

BAKER, G.J. Dental physiology. In: EASLEY, K.J.; BAKER, G.J. Equine dentistry. London: W.B. Saunders, 2002. p. 29-34.

CARMALT, J.L. Understanding the equine diastema. Equine Veterinary Education v. 15, n. 1, p. 34-35, 2003.

DIXON, P.M. The Gross, Histological, and Ultrastructural Anatomy of Equine Teeth and their Relationship to Disease. AAEP proceedings. v. 48, 2002.

DIXON, P.M.; TREMAINE, W.H.; PICKLES, K.; KUHNS, L.; HAWE, C.; MCCANN, J.; McGORUM, B.C.; RAILTON, D.I.; BRAMMER, S. Equine dental disease. Part 3: A long-term study of 400 cases: disorders of wear, traumatic damage and idiopathic fractures, tumours and miscellaneous disorders of the cheek teeth. Equine Veterinary Journal, v. 32, 2000.

DIXON, P.M.; DACRE, I.; KEMPSON, S. Idiopathic Cheek Teeth Fractures, Including Practice-based and Hospital-based Surveys. Proceedings of the American Association of Equine Practitioners, v. 52, 2006.

DIXON, P. M. A review of equine developmental disorders. Proceedings of the International Congress of the World Equine Veterinary Association, v. 12, 2011.

DIXON, P. M. Acquired Disorders of Equine Teeth. Proceedings of the American Association of Equine Practitioners - Focus Meeting, 2011.

EASLEY, J. Equine Dental Developmental Abnormalities. Proceedings of the em molares de cavalos de polo American Association of Equine Practitioners Annual Convention, v. 52, 2006.

ESALQ - Escola Superior de Agricultura Luiz de Queiroz. Estudo do Complexo do Agronegócio cavalo no Brasil. Brasília: CNA/ MAPA, 2006. 183 p.

FARIAS, S. K.; COUTINHO, G. M.; DONNER, A. C. Ocorrência de fraturas dentárias. Anais da Conferência Anual da Associação Brasileira dos Veterinários de Equídeos, v. 11, 2010.

HOLE, S.L.; DIXON, P.M. Equine dental disorders and occlusal pattern: Preliminary findings of a retrospective study (1999-2006). Proceedings of the British Equine Veterinary Association Congress, v. 47, p. 350,2008 dos animais foram identificadas com pelo menos uma, de 18 alterações odontológicas observadas.

As doenças mais prevalentes foram as pontas excessivas de esmalte dentário, edema de palato, lesões na mucosa jugal, seguidas pelas demais alterações do desgaste dentário.

Novos estudos são necessários para avaliar o impacto dessas alterações dentárias sobre a saúde geral e o escore corporal dos equinos ainda em vida, facilitando o dimensionamento do papel dessas lesões dentro do contexto do encaminhamento desses animais para o abate.

JOHNSON, J.T. Common Disorders of Incisor Teeth and Treatment. Proceedings of the American Association of Equine Practitioners Annual Convention, v. 52, 2006.

LANE, J.G. A review of dental disorders of the horse, their treatment and possible fresh approaches to management. Equine Veterinary Education, v. 6, p. 13-21, 1994.

LIMA, J.T.M.; ANDRADE, B.S.C.; SCHWARZBACH, S.V.; DE MARVAL, C.A.; LEAL, B.B.; FALEIROS, R.R.; ALVES, G.E.S. Ocorrência de doença infundibular, sobremordida e ganchos em equinos de cavalaria militar. Arquivos Brasileiros de Medicina Veterinária e Zootecnia, v. 63, n. 1, p. 6-11, 2011.

MUELLER, E.P.O. Equine dental disease: cause, diagnosis and treatment. Comp. North Am. Educ., v.13, p.1451-1461, 1991.

PAGLIOSA, G.M.; ALVES, G.E.S.; SCHWARZBACH, S.V. Odontologia Equina - Alterações dentárias frequentes que podem prejudicar a sanidade e performance do cavalo. Revista Brasileira de Medicina Equina, São Paulo, v. 5 n. 1, p. 7, 2006. Abnormalities in 483 Horses in the Netherlands. Principais afecções dentárias de equinos da mesorregião sul do Espírito Santo.

PETERS, J.W.E.; DE BOER, B.; VOORDE, G.B.M.B. Survey of Common Dental Proceedings of the American Association of Equine Practitioners Annual Convention, v. 52, 2006.

RIZZO, D.A.; LUCAS, F. A.; BARROS, R. J. S. Prevalência e diagnóstico das principais afecções dentárias de equinos da mesorregião sul do Espírito Santo. Pubvet, v. 5, n. 28, ed. 175, Art.1183, 2011. Association of Equine Practitioners Annual Convention.

RUCKER, B.A. Incisor procedures for field use. Proceedings of the American Association of Equine Practitioners, v. 42, p. 22-25, 1996.

RUCKER, B.A. Treatment of Equine Diastema. Proceedings of the American, v. 52, 2006.

SISSON, S.; GROSSMAN, J.D. Anatomia dos animais domésticos. Rio de Janeiro: Guanabara Koogan, 1986.

THOMASSIAN, A. Afecções do aparelho digestivo. In: THOMASSIAN, A. Enfermidade dos cavalos. 2. ed. São Paulo: Livraria Varela, 1990. p. 281-443.

WINTZER, H.J. et al. Doenças dos equinos: um manual para alunos e veterinários. São Paulo: Manole, 1990. 